\title{
Parameterized Modeling of Star Patterns for Traditional Latticed Screens
}

\author{
Vishal Gulati \\ GJ-University of Science and \\ Technology, Hisar, India
}

\author{
Puneet Katyal \\ GJ-University of Science and \\ Technology, Hisar, India
}

\begin{abstract}
This work makes the contribution of developing a parametric modeler for designing Islamic star patterns. The intention here is to innovate with new interpretations of star patterns for latticed screens, which are reminiscent of the Indo-Mughal era. With a motivation for providing traditional appearance to the buildings like resorts and hotels, traditional latticed screens with Islamic star patterns are used as a fundamental resource to archive decorative effects. Such latticed screens have been generated by using computer aided geometric modeling and manufacturing for commercial applications in the domain of architectural as well as interior decoration. Star patterns are realized as periodic and repeated arrangement of a type of motifs/compound-motifs. Further, motif/compound-motif is viewed as polar array of a primitive which is an atomic geometric object required for the representation of motif/compound-motif. Geometry of the primitives for motifs is defined in the form a planar map having a set of points. The positions of points are devised in terms of the modeling parameters and pairs of points are connected to generate a set of edges. By controlling the modeling parameters through a User Interface, patterns are rendered and further submitted to computer controlled laser cutting machine for fabrication.
\end{abstract}

\section{Keywords}

Islamic star pattern, latticed screen, motif, CAD

\section{INTRODUCTION}

Indian craft sector, which is growing by leaps and bounds, can be empowered with technological tools of design and manufacturing to keep this sector economically sustainable. Use of technological tools can not only give economic success by reducing drudgery and time involved in crafting, but also can provide opportunities for creative designing and innovative fabrication. Therefore, this work is directed towards the automation of craftsmanship by implementing CAD/CAM tools for producing real world crafted artifacts. For instance in this work, latticed screens, which are reminiscent of the Indo-Mughal era, have been regenerated by using computer aided design and manufacturing for commercial applications in the domain of architectural as well as interior decoration. With a motivation for providing traditional appearance to the buildings like resorts and hotels, traditional latticed screens with Islamic star patterns are used as a fundamental resource to archive decorative effects.

Latticed screens have been universally used in Indo-Mughal monuments on edges of platforms, causeways and terraces (as railings), and also on balconies. These have been employed gorgeously to close window and door openings for veil and ventilation. Moreover, latticed screen produces aesthetic effect in the room during the day as sunlight streams through it, the pattern forms magical shadow and graces the adjacent floors and walls (see Figure 1). Being such a useful architectural accessory, this work has laid emphasis on the decoration of latticed screens with Islamic art which mostly focus on the depiction of star patterns.
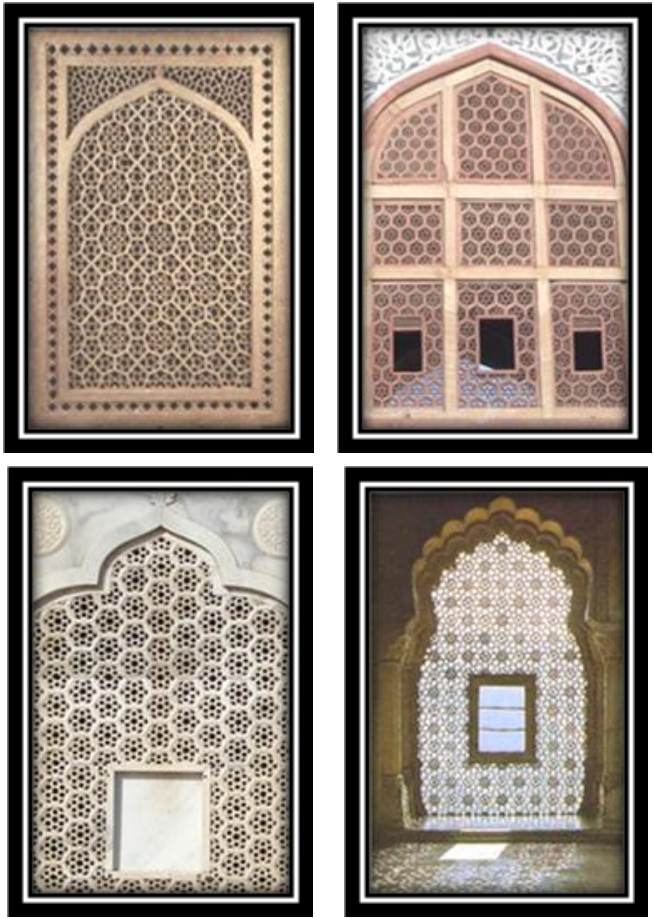

Figure 1: Latticed Screens Crafted during Indo-Mughal Period

The intention here is to innovate with new interpretations of star patterns for latticed screens. Traditional star patterns are understood as a set of motifs and relationships among them. In formal sense, this understanding corresponds to view a star pattern in terms of vocabulary of star motifs in the artifact, along with transformations rules between them. A variety of patterns can be generated with the appropriate definitions of motifs and rules.

In particular, this work makes the technical contribution of developing a parametric modeler for designing Islamic star patterns. A variety of star patterns can be rendered by defining modeling parameters. The rendered patterns are $2 \mathrm{D}$ in nature and ideally suited to computer-controlled manufacturing. Since traditional latticed screen is a plane pierced panel crafted out from one piece of stone/wood and adorned with regular and symmetric network of a pattern. Therefore, a rendered pattern is executed on a $2 \frac{1}{2} \mathrm{D}$ planar surface in a manufacturing environment and 
material other than the pattern is removed from one piece of material, thus leaving with a pierced network of a pattern.

A lot of methodologies have been created to reproduce Islamic star patterns [1-7]. Grunbaum and Shephard's derived a fundamental region by decomposing the star pattern by their symmetry groups (Figure 2a) [8-9]. Dewdney's gave a method of reflecting lines of periodically placed circles (Figure 2b) [10]. Hankin presented an algorithm for turning a tiling into a star pattern by joining the midpoints of the edges of the tiling (Figure 2c) $[11]$.

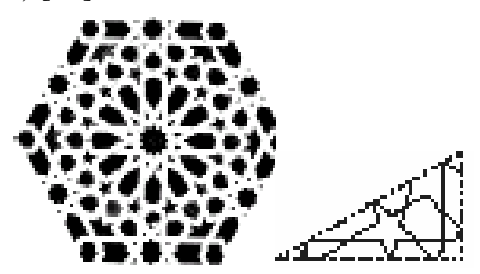

(a)

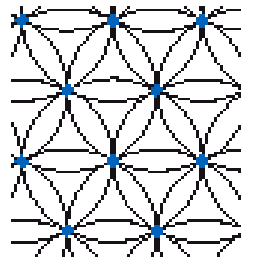

(b)

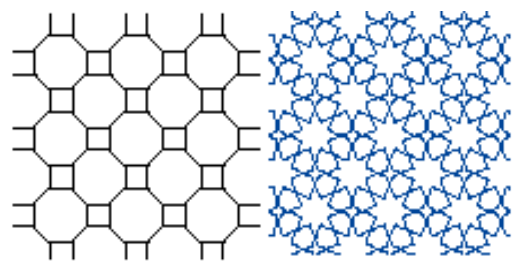

(c)

Figure 2: (a) Grunbaum and Shephard's Method (b) Dewdney's method (c) Hankin's method

\section{COMPREHENSION OF STAR PATTERNS FOR LATTICED SCREENS}

From symmetry point of view, all star patterns for traditional latticed screens (see Figure 3 ) have been realized as periodic and repeated arrangement of a type of compound-motifs. Further, a compound-motif is viewed as an array of different star/rosette motifs (Figure 4). Therefore, in this paradigm, patterns for latticed screens are generated with the description of three hierarchical levels which are labeled as: Motifs, Compound-motif and Pattern (Figure 4).

At the first level, the most characteristic motifs, stars and rosettes are designed. A star is regarded as a collection of line segments that are joined with each other at their end points. A rosette may be viewed as a star to which hexagons are attached in the concavities between adjacent points. These motifs may be enclosed within a boundary polygon.

At the next level, compound-motifs are designed with a theme of having a motif (star/rosette) surrounded by other motifs. The aesthetic sense of a compound motif has been derived from its geometrical symmetry. From aesthetic point of view, it is realized that three same or different types of motifs are positioned (one at centre and two at its surroundings) in a compound-motif. Same types of star motifs are placed at alternate positions around the central motif of having only even number of rays (Figure 4). From structure point of view, all compound-motifs are viewed as a connection of motifs where each of the surrounding motifs is in contact with the central motif and it's neighboring one. The connection is through the star/rosette rays and/or sides of polygon. This connection depends upon the algebraic sum of interior angles of boundary polygons of three motifs. If algebraic sum is $360^{\circ}$, only then each surrounded motif is in contact with its neighboring one and central motif. Such types of cases are represented in Table 1.

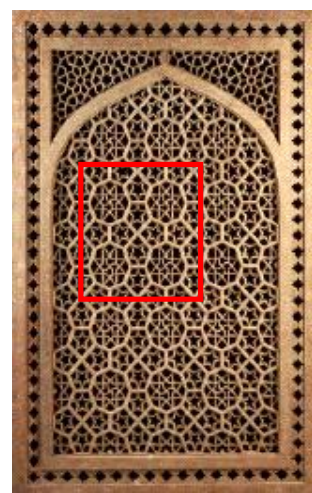

Pattern

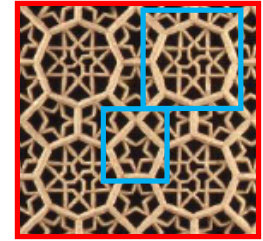

Compound-motif

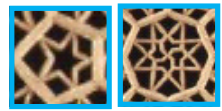

Motifs
Figure 3: Traditional Latticed Screen Crafted during Indo-Mughal Period of Akbar [12]

Lastly, patterns are generated with regular arrays of motifs/compound-motifs. These patterns are regarded as a planar map on a $2 \frac{1}{2} \mathrm{D}$ surface from which material other than line thicknesses is removed to manufacture the latticed screen. From manufacturing point of view, it is realized that the screens with star patterns can only be produced by cutting material out of one piece of planar surface because of its latticed structure.

\section{MODELING PARAMETERS}

Patterns for latticed screens take the form of a division of the surface into motifs/compound-motifs which are expressed as parameterized elements in order to provide a wide modeling range. The idea here is to create a rich source of star patterns by defining following intuitive modeling parameters (shown in Figure 5).

- Size of the motif equal to size of circumscribed boundary polygon (r)

- Number of star/rosette rays pointed towards the midpoint of all sides of the polygon (n)

- Shape of the star/rosette defined by angle of incidence/reflection of the star ray $(\theta)$

- Width of the rosette deciding its shape (w)

- Line thickness of the star/rosette rays and boundary polygon $\left(t_{1}\right.$ and $\left.t_{2}\right)$

- Number of rows and columns of motifs according to the size of the screen required( $R$ and $C$ )

- Arrangement of the compound-motifs/motifs in a pattern defined by row and column offset ( $\mathrm{RO}$ and $\mathrm{CO})$

\section{GEOMETRY OF THE PRIMITIVE FOR STAR MOTIFS/COMPOUND-MOTIFS}

The term primitive is used with a meaning of atomic geometric object required for the representation of star shaped 
motifs/compound-motifs. Primitive is described by modeling parameters. It is in the form a planar map having a set of points. The positions of points are devised in terms of the modeling parameters and pairs of points are connected to generate a set of edges in the planar map.

Geometry of the primitives for star shaped motifs and compound motifs created from a set of edges by joining the defined points is depicted in Figure 6 and Table 2. A star shaped motif/compoundmotif is viewed as a polar array of the primitives. Some of rendered star shaped motifs and compound-motifs are shown in Figure 7 (a \& c).

Using the same concept, geometry of the primitives for rosette shaped motifs and compound motifs have been found. Some of rendered star shaped motifs and compound-motifs are also shown in Figure 7 (b \& d).

\section{PATTERNS}

Patterns are composed of motifs which are multiplied and ordered in regular sequences. A rectangular array of motif is generated by defining the following parameters: (1) Number of rows (R) (2) Number of columns (C) (3) Row offset (RO) (4) Column offset (CO). The first two parameters are taken according to the size of the surface of the screen to be created. The row and column offsets parameters depend on the size of generated motif/compound. Some of rendered star/rosette shaped patterns are shown in Figure 8.

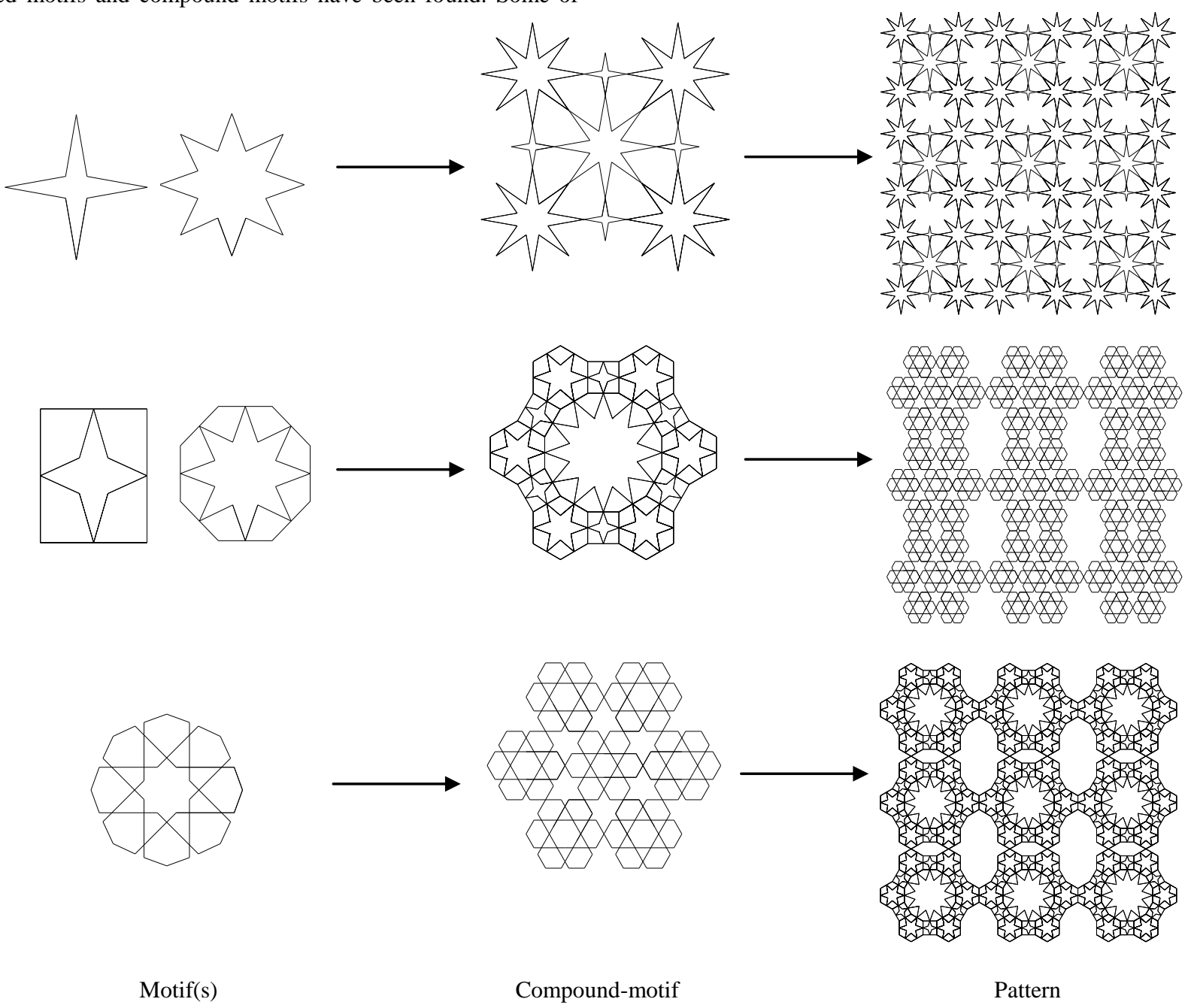

Figure 4: Patterns for Latticed Screens generated with Three Hierarchical Levels

\section{IMPLEMENTATION ANDVALIDATION}

This paradigm has been implemented under the ActiveX and Visual Basic Application (VBA) programming environment using AutoCAD. VBA runs in the same process space as AutoCAD which provides programmable control through the ActiveX Automation interface. By putting the modeling parameters through a User Interface (Figure 9), ornamental patterns are rendered and further submitted to computer controlled laser cutting machine for removing material out of single piece. Laser cutting utilizes the power of light to obtain precise cuts with minimal waste and leaves a clean finish. The depth of cut produced can be varied by laser cutting as it depends on the power of the light being focused through the laser lens. This work has been validated by fabricating the rendered patterns on a computer controlled laser cutting machine (Figure 10). 
Table 1: Cases when Each Surrounding Motif is in Contact with Central Motif and its Neighboring one

\begin{tabular}{|c|c|c|}
\hline $\begin{array}{c}\text { Sr. } \\
\text { No. }\end{array}$ & $\begin{array}{c}\text { Interior angles of polygons } \\
\text { of three motifs used in a } \\
\text { compound-motif (algebraic } \\
\left.\text { sum }=360^{\circ}\right)\end{array}$ & $\begin{array}{c}\text { Number of rays of } \\
\text { star/rosette of three motifs } \\
\text { used in a compound-motif }\end{array}$ \\
\hline 1. & $120^{\circ}, 120^{\circ}, 120^{\circ}$ & $6,6,6$ \\
\hline 2. & $135^{\circ}, 135^{\circ}, 90^{\circ}$ & $8,8,4$ \\
\hline 3. & $144^{\circ}, 108^{\circ}, 108^{\circ}$ & $10,5,5$ \\
\hline 4. & $144^{\circ}, 156^{\circ}, 60^{\circ}$ & $10,15,3$ \\
\hline 5. & $150^{\circ}, 150^{\circ}, 60^{\circ}$ & $12,12,3$ \\
\hline 6. & $150^{\circ}, 120^{\circ}, 90^{\circ}$ & $12,6,4$ \\
\hline 7. & $160^{\circ}, 140^{\circ}, 60^{\circ}$ & $18,9,3$ \\
\hline 8. & $162^{\circ}, 108^{\circ}, 90^{\circ}$ & $20,5,4$ \\
\hline 9. & $165^{\circ}, 135^{\circ}, 60^{\circ}$ & $24,8,3$ \\
\hline
\end{tabular}
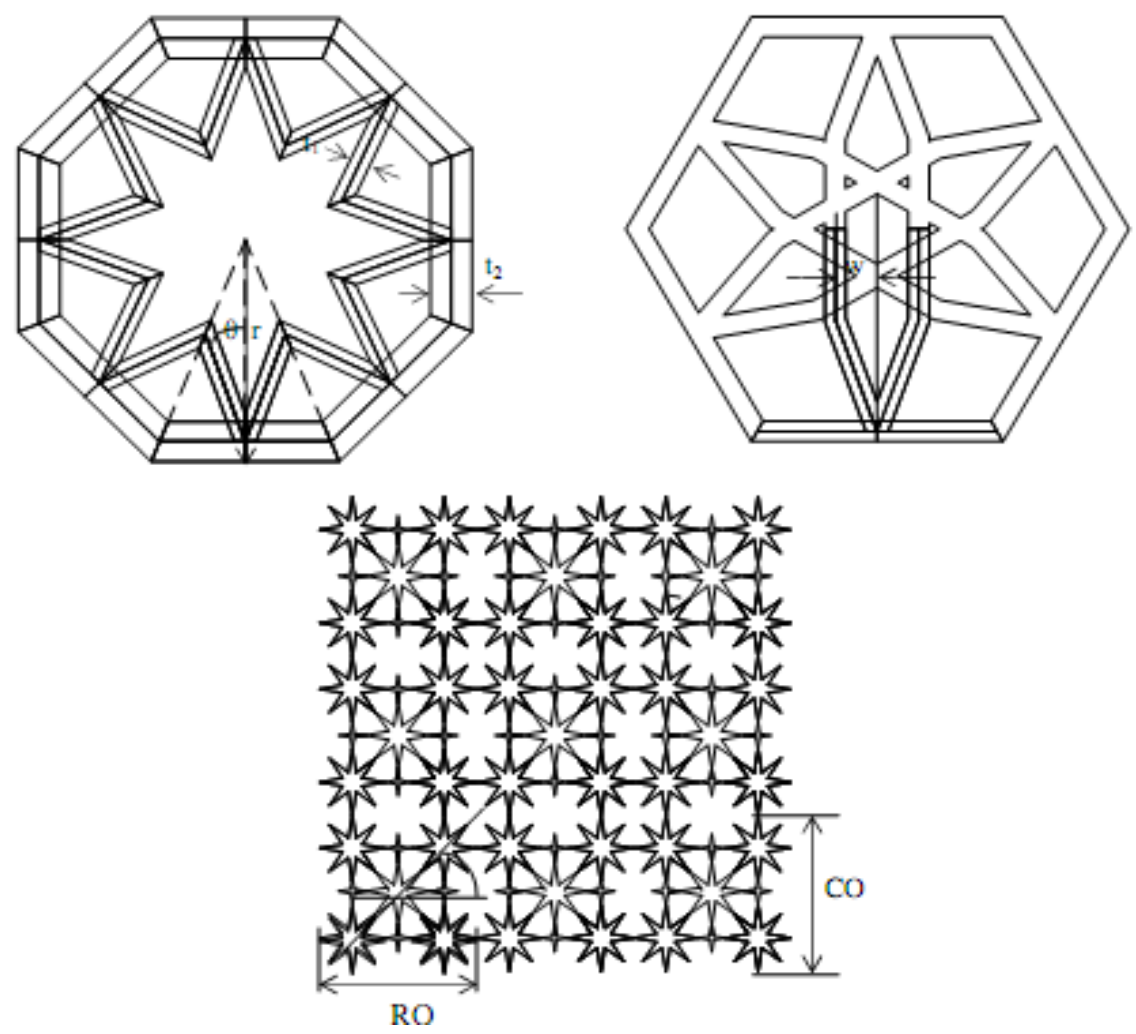

Figure 5: Modeling Parameters 


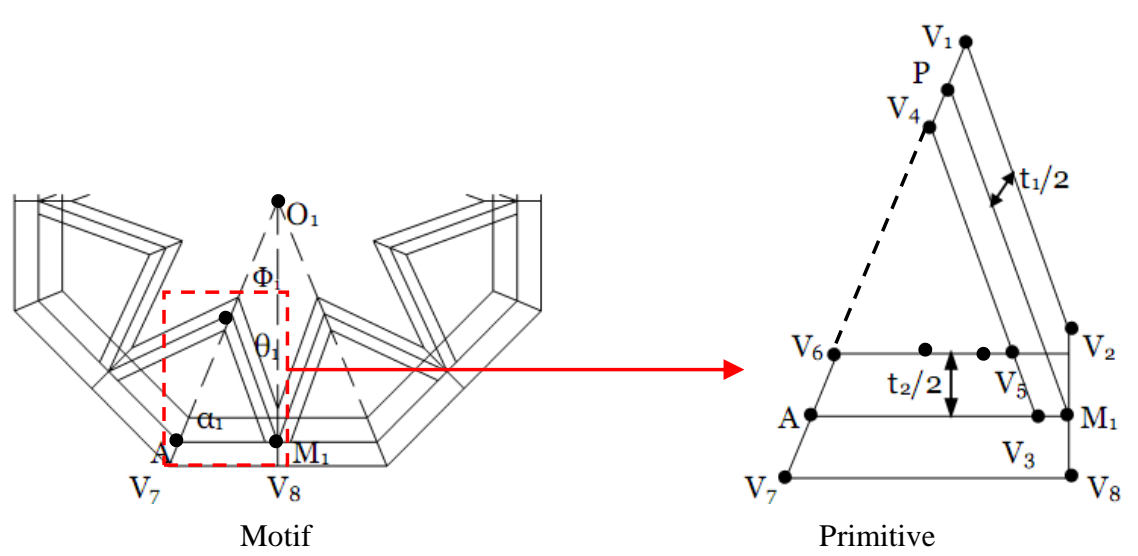

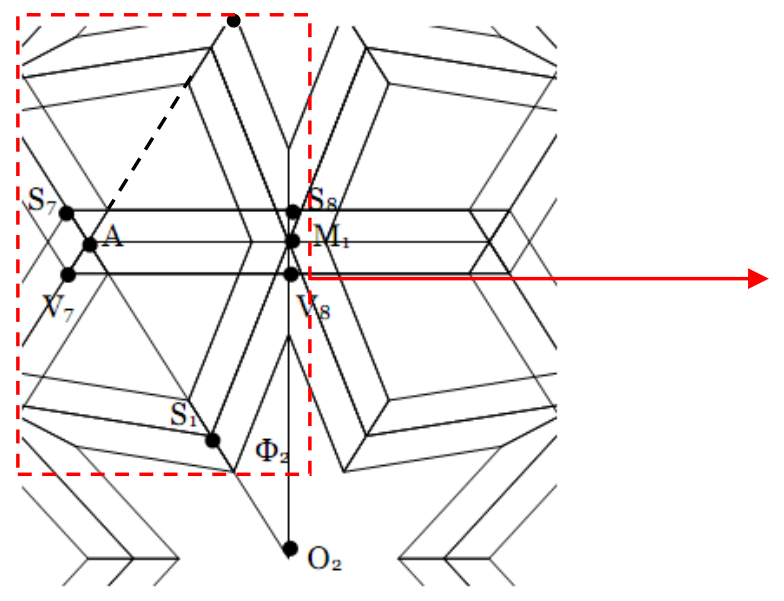

Compound-motif

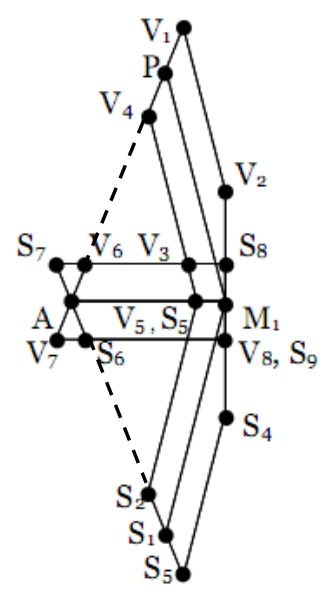

Primitive

Figure 6: Geometry of the Primitives for Star Shaped Motifs and Compound-motifs

Table 2: Set of Devised Points in the Primitives of Star Shaped Motifs and Compound-motifs

\begin{tabular}{|c|c|c|}
\hline Points & Position of the Points & Remarks \\
\hline$O_{1}$ & $X_{o_{1}}=0 \quad Y_{o_{1}}=0$ & Center point of boundary polygon \\
\hline$A$ & $\begin{array}{l}X_{A}=-r_{1} \tan \phi_{1} \quad Y_{A}=-r_{1} \\
\text { where } \quad \phi_{1}=\Pi / n\end{array}$ & \\
\hline$M_{1}$ & $X_{M_{1}}=0 \quad Y_{M_{1}}=-r_{1}$ & \\
\hline$P$ & $\begin{array}{l}X_{P}=-\sqrt{\left(P O_{1}\right)^{2}+\left(Y_{P}\right)^{2}} \quad Y_{P}=\frac{-\left(P O_{1}\right)^{2}+\left(P M_{1}\right)^{2}-r_{1}^{2}}{2 r_{1}} \\
\text { where } \quad P M_{1}=\frac{r_{1} \sin \phi_{1}}{\sin \left(\phi_{1}+\theta_{1}\right)} \quad P O_{1}=\frac{r_{1} \sin \theta_{1}}{\sin \left(\phi_{1}+\theta_{1}\right)}\end{array}$ & $\begin{array}{l}\mathrm{P} \text { is located on } \mathrm{AO}_{1} \text { such that angle } \mathrm{PM}_{1} \mathrm{O}_{1} \text { is } \theta_{1} \\
\text { (angle of incidence of the star ray) }\end{array}$ \\
\hline$V_{1}$ & $\begin{array}{l}X_{V_{1}}=\frac{-X_{P}+t_{1} \sin \phi_{1}}{2 \cos \left(\alpha_{1}-\theta_{1}\right)} \quad Y_{V_{1}}=\frac{-Y_{P}+t_{1} \cos \phi_{1}}{2 \cos \left(\alpha_{1}-\theta_{1}\right)} \\
\text { where } \quad \alpha_{1}=\frac{\Pi\left(n_{1}-2\right)}{2 n_{1}}\end{array}$ & $\mathrm{~V}_{1}$ is located on $\mathrm{AO}_{1}$ \\
\hline
\end{tabular}




\begin{tabular}{|c|c|c|}
\hline$V_{2}$ & $X_{V_{2}}=0 \quad Y_{V_{2}}=-r_{1}+\frac{t_{1}}{2 \sin \theta_{1}}$ & $\begin{array}{l}V_{2} \text { is located on } \mathrm{M}_{1} \mathrm{O}_{1} \text { such that distance between } \\
\text { edges } \mathrm{PM}_{1} \text { and } \mathrm{V}_{2} \mathrm{~V}_{1} \text { remains } \mathrm{t}_{1} / 2\end{array}$ \\
\hline$V_{3}$ & $X_{V_{3}}=-\frac{t_{1}}{2 \cos \theta_{1}} \quad Y_{V_{3}}=-r_{1}$ & $\mathrm{~V}_{3}$ is located on $\mathrm{AM}_{1}$ \\
\hline$V_{4}$ & $X_{V_{4}}=\frac{-X_{P}-t_{1} \sin \phi_{1}}{2 \cos \left(\alpha_{1}-\theta_{1}\right)} \quad Y_{V_{4}}=\frac{-Y_{P}-t_{1} \cos \phi_{1}}{2 \cos \left(\alpha_{1}-\theta_{1}\right)}$ & $\begin{array}{l}\mathrm{V}_{4} \text { is located on } \mathrm{AO}_{1} \text { such that distance between } \\
\text { edges } \mathrm{M}_{1} \mathrm{P} \text { and } \mathrm{V}_{3} \mathrm{~V}_{4} \text { remains } \mathrm{t}_{1} / 2\end{array}$ \\
\hline$V_{5}$ & $X_{V_{5}}=0 \quad Y_{V_{5}}=-r_{1}+\frac{t_{2}}{2}$ & $\mathrm{~V}_{5}$ is located on $\mathrm{MO}_{1}$ edge when extended \\
\hline$V_{6}$ & $X_{V_{6}}=-r_{1} \tan \phi_{1}+\frac{t_{2}}{2 \tan \alpha_{1}} \quad Y_{V_{6}}=-r_{1}+\frac{t_{2}}{2}$ & $\begin{array}{l}V_{6} \text { is located on } A_{1} \text { such that distance between } \\
\text { edges } M_{1} A \text { and } V_{5} V_{6} \text { remains } t_{2} / 2\end{array}$ \\
\hline$V_{7}$ & $X_{V_{7}}=-r_{1} \tan \phi_{1}-\frac{t_{2}}{2 \tan \alpha_{1}} \quad Y_{V_{7}}=-r_{1}+\frac{t_{2}}{2}$ & $\mathrm{~V}_{7}$ is located on $\mathrm{AO}_{1}$ \\
\hline$V_{8}$ & $X_{V_{8}}=0 \quad Y_{V_{8}}=-r_{1}-\frac{t_{2}}{2}$ & $\begin{array}{l}V_{8} \text { is located on } M_{1} O_{1} \text { such that distance between } \\
\text { edges } M_{1} A \text { and } V_{7} V_{8} \text { remains } t_{2} / 2\end{array}$ \\
\hline $\mathrm{O}_{2}$ & $\begin{array}{l}X_{o_{2}}=-r_{1}-r_{2} \quad Y_{o_{2}}=0 \\
\text { where } \quad r_{2}=\frac{r_{1} \tan \left(\phi_{1}\right)}{\tan \left(\phi_{2}\right)} \quad \phi_{2}=\prod / n_{2}\end{array}$ & Center point of a surrounded boundary polygon. \\
\hline$S_{1}$ & $\begin{array}{l}X_{S_{1}}=-\sqrt{\left(S_{1} O_{2}\right)^{2}+\left(Y_{S_{1}}\right)^{2}} \quad Y_{S_{1}}=\frac{-\left(S_{1} M_{1}\right)^{2}+\left(S_{1} O_{2}\right)^{2}-r_{2}^{2}-2 r_{1} r_{2}}{2 r_{2}} \\
\text { where } \quad S_{1} M_{1}=\frac{r_{2} \sin \left(\phi_{2}\right)}{\sin \left(\phi_{2}+\theta_{2}\right)} \quad S_{1} O_{2}=\frac{r_{2} \sin \left(\theta_{2}\right)}{\sin \left(\phi_{2}+\theta_{2}\right)}\end{array}$ & $\mathrm{S}_{1}$ is located on $\mathrm{AO}_{2}$ such that angle $\mathrm{S}_{1} \mathrm{M}_{1} \mathrm{O}_{2}$ is $\theta_{2}$ \\
\hline$S_{2}$ & $\begin{array}{l}X_{S_{2}}=\frac{-X_{S_{1}}-t_{1} \sin \left(\phi_{2}\right)}{2 \cos \left(\alpha_{2}-\theta_{2}\right)} \quad Y_{S_{2}}=\frac{-Y_{S_{1}}+t_{1} \cos \left(\phi_{2}\right)}{2 \cos \left(\alpha_{2}-\theta_{2}\right)} \\
\text { where } \quad \alpha_{2}=\frac{\Pi\left(n_{2}-2\right)}{2 n_{2}}\end{array}$ & $\mathrm{~S}_{2}$ is located on $\mathrm{AO}_{2}$ \\
\hline$S_{5}$ & $X_{S_{5}}=-\frac{t_{1}}{2 \cos \left(\theta_{2}\right)} \quad Y_{S_{5}}=-r_{1}$ & $\begin{array}{l}S_{5} \text { is located on } A_{1} \text { such that distance between } \\
\text { edges } S_{1} M_{1} \text { and } S_{2} S_{5} \text { remains } t_{1} / 2\end{array}$ \\
\hline$S_{3}$ & $X_{S_{3}}=\frac{-X_{S_{1}}+t_{1} \sin \left(\phi_{2}\right)}{2 \cos \left(\alpha_{2}-\theta_{2}\right)} \quad Y_{S_{3}}=\frac{-Y_{S_{1}}-t_{1} \cos \left(\phi_{2}\right)}{2 \cos \left(\alpha_{2}-\theta_{2}\right)}$ & $\mathrm{S}_{3}$ is located on $\mathrm{AO}_{2}$ \\
\hline$S_{4}$ & $X_{S_{4}}=0 \quad Y_{S_{4}}=-r_{1}-\frac{t_{1}}{2 \sin \left(\theta_{2}\right)}$ & $\begin{array}{l}\mathrm{S}_{4} \text { is located on } \mathrm{O}_{2} \mathrm{M}_{1} \text { such that distance between } \\
\text { edges } \mathrm{S}_{1} \mathrm{M}_{1} \text { and } \mathrm{S}_{2} \mathrm{~S}_{5} \text { remains } \mathrm{t}_{2} / 2\end{array}$ \\
\hline$S_{6}$ & $X_{S_{7}}=-r_{1} \tan \left(\phi_{2}\right)+\frac{t_{2}}{2 \tan \left(\alpha_{2}\right)} \quad Y_{S_{7}}=-r_{1}-\frac{t_{2}}{2}$ & $\mathrm{~S}_{6}$ is located on $\mathrm{AO}_{2}$ \\
\hline$S_{9}$ & $X_{S_{9}}=0 \quad Y_{S_{9}}=-r_{1}-\frac{t_{2}}{2}$ & $\begin{array}{l}\mathrm{S}_{9} \text { is located on } \mathrm{O}_{2} \mathrm{M}_{1} \text { such that distance between } \\
\text { edges } \mathrm{AM}_{1} \text { and } \mathrm{S}_{6} \mathrm{~S}_{9} \text { remains } t_{2} / 2\end{array}$ \\
\hline$S_{7}$ & $X_{S_{7}}=-r_{1} \tan \left(\phi_{2}\right)-\frac{t_{2}}{2 \tan \left(\alpha_{2}\right)} \quad Y_{S_{7}}=-r_{1}+\frac{t_{2}}{2}$ & $\mathrm{~S}_{7}$ is located on extended edge $\mathrm{AO}_{2}$ \\
\hline$S_{8}$ & $X_{S_{8}}=0 \quad Y_{S_{8}}=-r_{1}+\frac{t_{2}}{2}$ & $\begin{array}{l}\mathrm{S}_{8} \text { is located on } \mathrm{O}_{2} \mathrm{M}_{1} \text { such that distance between } \\
\text { edges } \mathrm{AM}_{1} \text { and } \mathrm{S}_{7} \mathrm{~S}_{8} \text { remains } t_{2} / 2\end{array}$ \\
\hline
\end{tabular}




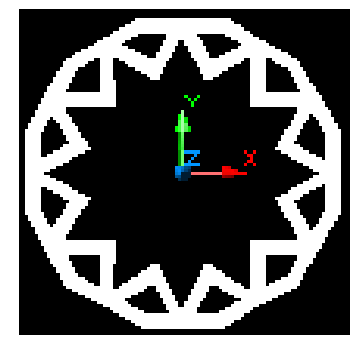

$\mathrm{r}_{1}=1, \mathrm{t}_{1}=0.1, \mathrm{t}_{2}=0.1$, $\mathrm{n}_{1}=12, \theta_{1}=30^{\circ}$

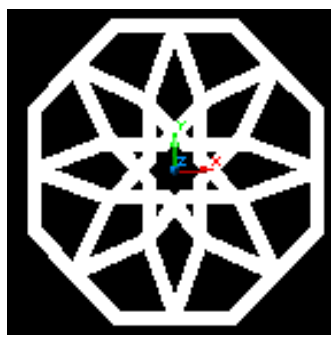

$\mathrm{r}_{1}=1, \mathrm{t}_{1}=0.1, \mathrm{t}_{2}=0.1$ $\mathrm{n}_{1}=8, \theta_{1}=20^{\circ}, \mathrm{w}_{1}=0.18$

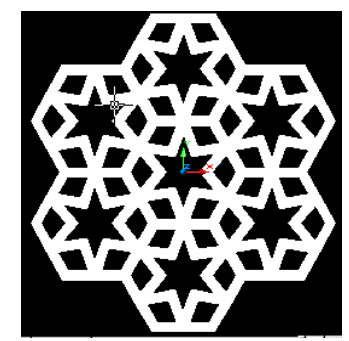

$\mathrm{r}_{1}=1, \mathrm{t}_{1}=0.2, \mathrm{t}_{2}=0.2, \mathrm{n}_{1}=6$, $\theta_{1}=20^{\circ}, \mathrm{n}_{2}=6, \theta_{2}=20^{\circ}, \mathrm{n}_{3}=6$, $\theta_{3}=20^{\circ}$

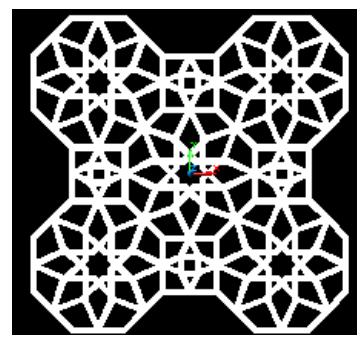

$\mathrm{r}_{1}=1, \mathrm{t}_{1}=0.1, \mathrm{t}_{2}=0.1, \mathrm{n}_{1}=8, \theta_{1}=20^{\circ}$,

$\mathrm{w}_{1}=0.18, \mathrm{n}_{2}=4, \theta_{2}=20^{\circ}, \mathrm{w}_{2}=0.18$, $\mathrm{n}_{3}=8, \theta_{3}=20^{\circ}, \mathrm{w}_{3}=0.18$

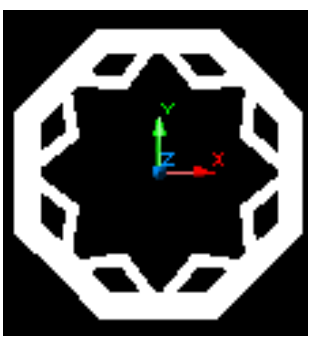

$\mathrm{r}_{1}=1, \mathrm{t}_{1}=0.1, \mathrm{t}_{2}=0.2$,

$\mathrm{n}_{1}=8, \theta_{1}=30^{\circ}$

(a) Star Shaped Motifs

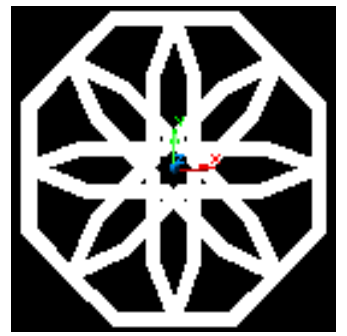

$\mathrm{r}_{1}=1, \mathrm{t}_{1}=0.1, \mathrm{t}_{2}=0.1$,

$\mathrm{n}_{1}=8, \theta_{1}=20^{\circ}, \mathrm{w}_{1}=0.14$

(b) Rosette Shaped Motifs

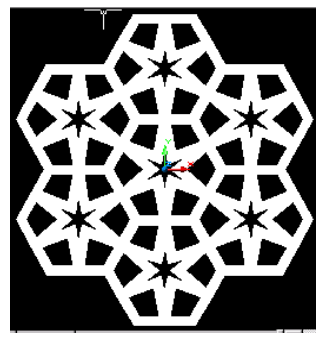

$\mathrm{r}_{1}=1, \mathrm{t}_{1}=0.2, \mathrm{t}_{2}=0.2, \mathrm{n}_{1}=6$,

$\theta_{1}=10^{\circ}, \mathrm{n}_{2}=6, \theta_{2}=10^{\circ}, \mathrm{n}_{3}=6$, $\theta_{3}=10^{\circ}$

(c) Star Shaped Compound-motifs

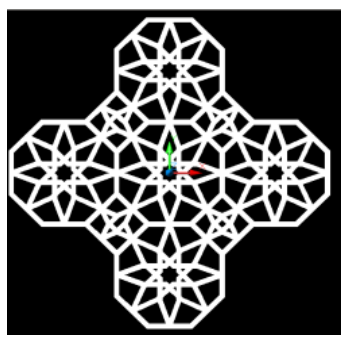

$\mathrm{r}_{1}=1, \mathrm{t}_{1}=0.1, \mathrm{t}_{2}=0.1, \mathrm{n}_{1}=8, \theta_{1}=20^{\circ}$, $\mathrm{w}_{1}=0.18, \mathrm{n}_{2}=8, \theta_{2}=20^{\circ}, \mathrm{w}_{2}=0.18$, $\mathrm{n}_{3}=4, \theta_{3}=20^{\circ}, \mathrm{w}_{3}=0.18$

(d) Rosette Shaped Compound-motifs

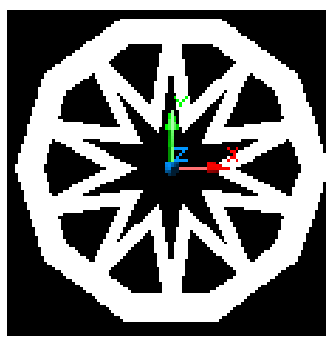

$\mathrm{r}_{1}=1, \mathrm{t}_{1}=0.1, \mathrm{t}_{2}=0.2$, $\mathrm{n}_{1}=10, \theta_{1}=10^{\circ}$

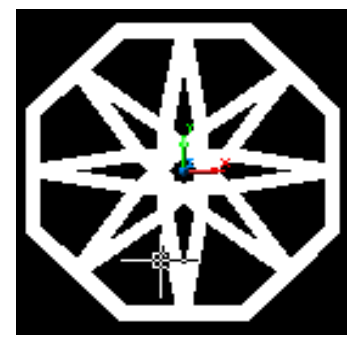

$\mathrm{r}_{1}=1, \mathrm{t}_{1}=0.1, \mathrm{t}_{2}=0.1$, $\mathrm{n}_{1}=8, \theta_{1}=10^{\circ}, \mathrm{w}_{1}=0.12$

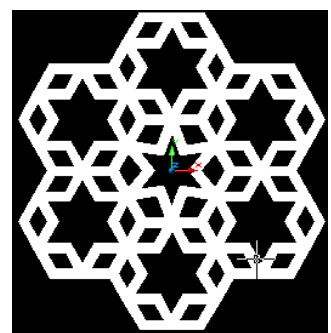

$\mathrm{r}_{1}=1, \mathrm{t}_{1}=0.2, \mathrm{t}_{2}=0.2, \mathrm{n}_{1}=6$, $\theta_{1}=30^{\circ}, \mathrm{n}_{2}=6, \theta_{2}=30^{\circ}, \mathrm{n}_{3}=6$, $\theta_{3}=10^{\circ}$

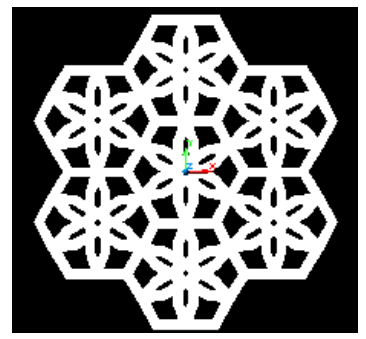

$\mathrm{r}_{1}=1, \mathrm{t}_{1}=0.1, \mathrm{t}_{2}=0.1, \mathrm{n}_{1}=6, \theta_{1}=20^{\circ}$, $\mathrm{w}_{1}=0.15, \mathrm{n}_{2}=6, \theta_{2}=20^{\circ}, \mathrm{w}_{2}=0.15$, $\mathrm{n}_{3}=6, \theta_{3}=20^{\circ}, \mathrm{w}_{3}=0.15$

Figure 7: Rendered Motifs and Compound-Motifs 


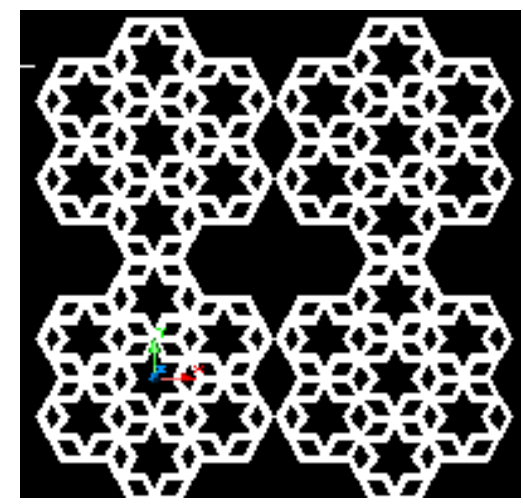

$\mathrm{r}_{1}=1, \mathrm{t}_{1}=0.2, \mathrm{t}_{2}=0.2, \mathrm{n}_{1}=6, \theta_{1}=20^{\circ}$ $\mathrm{n}_{2}=6, \theta_{2}=20^{\circ}, \mathrm{n}_{3}=6, \theta_{3}=20^{\circ}, \mathrm{R}=2$, $\mathrm{C}=2, \mathrm{RO}=4.8284, \mathrm{CO}=4.8284$

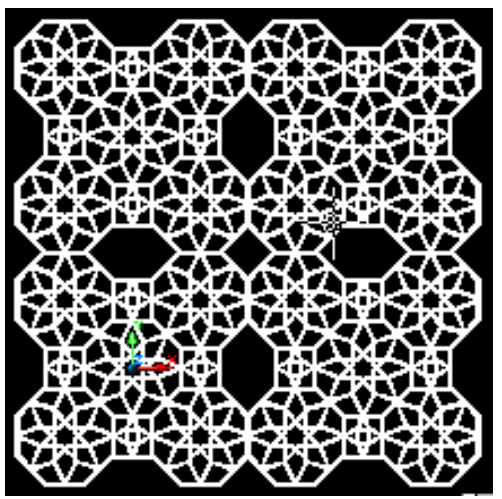

$\mathrm{r}_{1}=1, \mathrm{t}_{1}=0.2, \mathrm{t}_{2}=0.2, \mathrm{n}_{1}=8, \theta_{1}=20^{\circ}$ $\mathrm{w}_{1}=0.18, \mathrm{n}_{2}=4, \theta_{2}=20^{\circ}, \mathrm{w}_{2}=0.18$, $\mathrm{n}_{3}=8, \theta_{3}=20^{\circ}, \mathrm{w}_{3}=0.18, \mathrm{R}=2, \mathrm{C}=2$, $\mathrm{RO}=5.889, \mathrm{CO}=6.0$

Figure 8: Star/Rosette Shaped Patterns

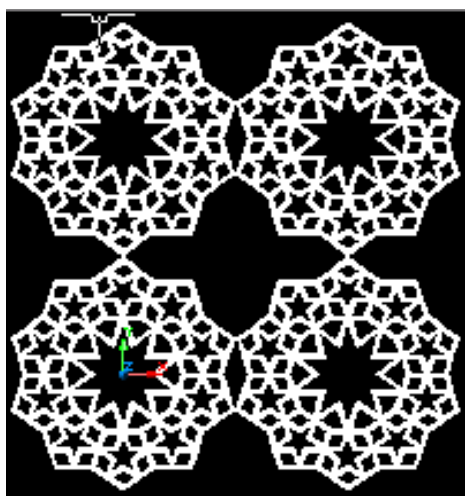

$\mathrm{r}_{1}=1, \mathrm{t}_{1}=0.2, \mathrm{t}_{2}=0.2, \mathrm{n}_{1}=10$,

$\theta_{1}=20^{\circ}, \mathrm{n}_{2}=5, \theta_{2}=20^{\circ}, \mathrm{n}_{3}=5$,

$\theta_{3}=20^{\circ}, \mathrm{R}=2, \mathrm{C}=2, \mathrm{RO}=4.1236$, $\mathrm{CO}=3.9218$

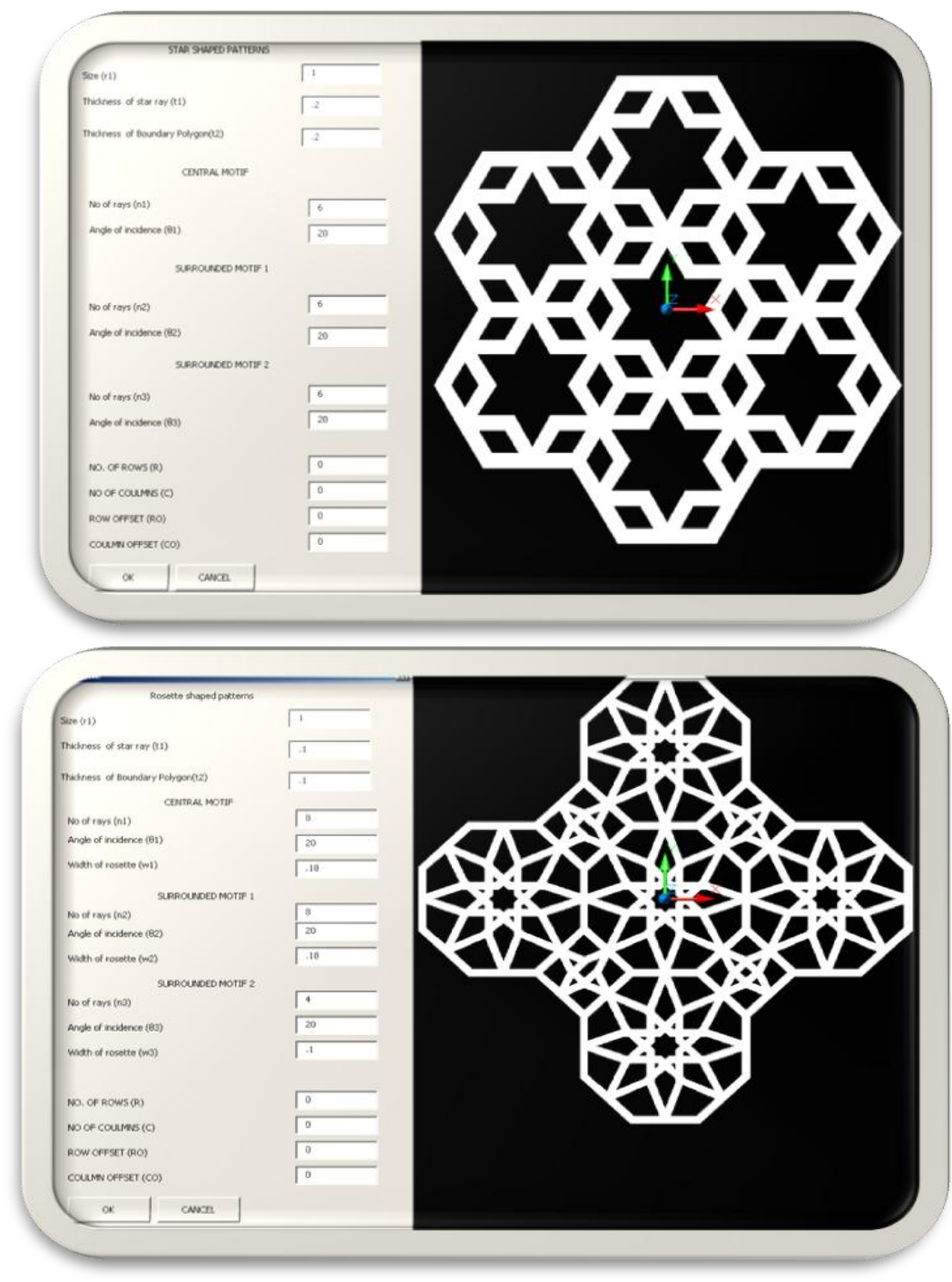

Figure 9: Rendered Patterns by Putting Modeling Parameters through User Interface 

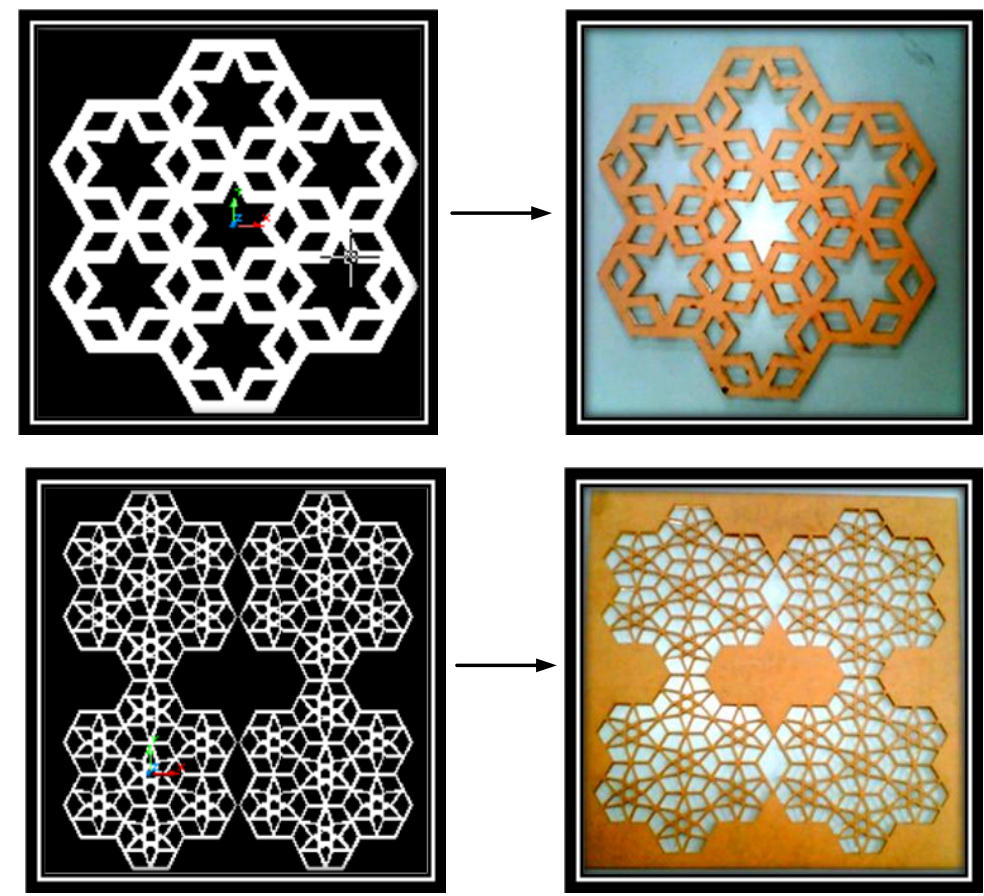

Figure 10: CAD Models (Left) and Actual Patterns Cut by Laser Cutting Machine (Right)

\section{CONCLUDING REMARKS}

This work presents a modeler to develop an artifact in the domain of architecture as well as interior decoration. The idea is to use the field of computer aided geometric modeling and manufacturing towards product representation. The proposed modeler produces computer understandable models that support diverse integrated manufacturing. Moreover, it possesses a wide modeling range i.e. they are able to generate a wide range of design models. As latticed screen is not only a functional architectural product, but a decorative item, proposed modeler is capable of representing complete ornamental product and not just shapes. It also supports a variety of manufacturing applications such as CNC milling, laser cutting and engraving and waterjet cutting.

\section{REFERENCES}

1. Bourgoin J., 1973, Arabic Geometrical Pattern and Design, Dover Publications.

2. Atallah M. J., 1985, On Symmetry Detection, IEEE Trans. Computers, 34, 663-666.

3. Abas S. J. and Salman A. S., 1992, Geometric and GroupTheoretic Methods for Computer Graphics Studies of Islamic
Symmetric Patterns, Computer Graphics Forum, 11(1), 4353.

4. Castera J. M., 1999, Zellijs, Muqarnas and Quasicrystals, ISAMA 99 Proceedings, 99-104.

5. Kaplan C. S. 2002, Computer Graphics and Geometric Ornamental Design, Ph.D., University of Washington, Seattle.

6. Bonner J., 2003, Three Traditions of Self-Similarity in Fourteenth and Fifteenth Century Islamic Geometric Ornament, In Reza Sarhangi and Nathaniel Friedman, ISAMA/BRIDGES Proceedings, 1-12.

7. Kaplan C. S. and Salesin D. H., 2004, Islamic Star Patterns in Absolute Geometry, ACM Transactions on Graphics, 23.

8. Grünbaum B. and Shephard G. C., 1987, Tilings and Patterns, W. H. Freeman.

9. Grünbaum B. and Shephard G. C., 1992, Interlace Patterns in Islamic and Moorish art, Leonardo, 25, 331-339.

10. Dewdney A.K., 1993, The Tinkertoy Computer and Other Machinations, 222-230.

11. Hankin E. H., 1925, The Drawing of Geometric Patterns in Saracenic Art, Memoirs of the Archaeological Society of India, Government of India.

12. Jali Screen, http://www.metamuseum.org. 